\title{
Ideología en prensa española: tratamiento discursivo de la enfermedad rara ataxia
}

\author{
Susana RIDAO RoDRIGO \\ Universidad de Murcia \\ sridao@um.es
}

Recibido: 21/01/2011

Aceptado: 22/07/2011

\begin{abstract}
Resumen
La prensa constituye un medio idóneo para analizar la ideología subyacente en el tratamiento comunicativo de determinados aspectos sociales. Los trabajos existentes en este terreno se han centrado, prioritariamente, en investigar la inmigración; en nuestra opinión, esta situación es, en cierto modo, extrapolable al tratamiento de enfermedades con baja prevalencia. Por ello, en este artículo realizamos un estudio de la representación discursiva de la ataxia, una enfermedad rara, en la prensa española; más en concreto, analizamos los diarios El País, El Mundo y $A B C$, a partir de variables cuantitativas y cualitativas. Entre las conclusiones aportadas destacamos que, como era de esperar, estas noticias tratan asuntos sanitarios; no obstante, tan solo el $36.08 \%$ del corpus obtenido presenta la enfermedad de la ataxia como tema prioritario.
\end{abstract}

Palabras clave: Ideología, representación discursiva, análisis crítico del discurso, enfermedades raras y ataxia.

\section{Ideology in Spanish Press: Discursive Treatment of Rare Disease Ataxia}

\begin{abstract}
The press is a suitable means to analyze the underlying ideology in the treatment of certain social aspects of communication. Existing publications in this area have focused, primarily, on researching in immigration; in our opinion, this situation is, in some way, extrapolated to treatment of diseases with low prevalence. Thus, this paper conducted a study of the discursive representation of ataxia, a rare disease, in the Spanish press; more specifically, we analyze the newspapers El Pais, El Mundo and ABC, using quantitative and qualitative variables. As our foremost conclusion, we would like to emphasize that, as expected, these news deal with health issues, but only the $36.08 \%$ of the corpus obtained approach the disease of ataxia as a priority topic.
\end{abstract}

Keywords: Ideology, discursive representation, Critical Discourse Analysis, rare diseases and ataxia.

\section{Referencia normalizada}

RIDAO RODRIGO, Susana (2011): "Ideología en prensa española: tratamiento discursivo de la enfermedad rara ataxia". Estudios sobre el mensaje periodístico. Vol. 17, núm. 2, págs.: 581-594. Madrid, Servicio de Publicaciones de la Universidad Complutense.

Sumario: 1. Introducción: el panorama de las enfermedades raras. 2. Marco teórico. 3. Corpus y metodología. 4. Resultados. 4. 1. Datos cuantitativos. 4. 2. Datos cualitativos. 5. Discusión. 5. 1. Datos cuantitativos. 5. 2. Datos cualitativos. 6. Conclusiones. 7. Referencias bibliográficas.

\section{Introducción: el panorama de las enfermedades raras}

En la última década en España, sobre todo a raíz del nacimiento y la expansión de las actividades llevadas a cabo por FEDER (Federación Española de Enfermedades Raras), las patologías poco prevalentes están dándose a conocer. La Organización Mundial de la Salud establece que una enfermedad debe considerarse rara cuando el índice de frecuencia que presenta es inferior a un individuo por cada dos mil habitantes. Además, se estima que existen entre cinco y siete mil tipologías de enferme- 
dades raras. Siguiendo con los datos cuantitativos, según FEDER, en España tenemos unos tres millones de afectados por estas patologías (AUTORES VARIOS, 2009). Tales cifras contrastan con la incomunicación existente en torno a estas enfermedades. Aclaremos lo dicho: durante mucho tiempo los pacientes aquejados por patologías poco prevalentes han carecido de visibilidad, a pesar de ser un grupo numeroso (MATEO, 2001 y GONZÁLEZ-MENESES et alii, 2008).

Aunque en los últimos años las enfermedades raras aparecen con mediana frecuencia en los medios, hemos de señalar que la sociedad, en general, desconoce su existencia. Esta situación se vuelve terriblemente perversa cuando observamos que en determinadas ocasiones ni siquiera el personal sanitario está al día de estas patologías (ENSERio, 2009). Es cierto que el elevado número de las mismas hace que diagnosticarlas se convierta en una ardua tarea, y que con frecuencia haya pacientes con un diagnóstico, y en consecuencia un tratamiento, equivocado. En estos momentos, se están llevando a cabo investigaciones en ciertas patologías, si bien hemos de señalar que se trata de casos aislados. La política económica imperante en el sistema capitalista mundial obstaculiza la investigación en este campo, pues la nula o escasa rentabilidad económica que aportaría la incorporación al mercado de fármacos que pudieran paliar los síntomas de estas enfermedades hace que los laboratorios farmacéuticos los principales promotores de este tipo de proyectos- no se interesen por los mismos.

Cada vez con una frecuencia más elevada en los medios de comunicación aparecen noticias sobre enfermedades raras, en general, o determinadas patologías poco prevalentes, en particular. En este trabajo nos proponemos analizar la representación discursiva de la ataxia en los diarios El País, El Mundo y $A B C$. Dando una visión muy simplista, la ataxia (cuya etimología griega significa sin orden) es una enfermedad que afecta a la coordinación del movimiento que comporta una desviación del segmento corporal o segmentos corporales que lo están realizando, de la línea ideal que debe seguir durante su recorrido. Esto implica que un enfermo de ataxia presenta titubeos al mover las piernas y los brazos (OBSERVATORIO DE LA DiSCAPACIDAD, 2002: 13). Aunque la más común es la ataxia de Friedrich, existen más de quinientas tipologías distintas de ataxia.

La soledad que ha caracterizado a las enfermedades raras hasta el siglo XX, a pesar de que es cierto que ya en el siglo XXI está cobrando mayor protagonismo, ha repercutido directamente en la producción bibliográfica existente. Dentro del relativamente escaso número de trabajos publicados sobre el tema, la mayor parte de tales aportaciones ha sido enfocada desde perfiles principalmente sanitarios. Por supuesto, en el panorama anglosajón podemos encontrar bibliografía muy interesante sobre los aspectos comunicativos en los ámbitos sanitarios -citemos auténticos clásicos como SONTAG (1978), LuCy (1997), GWYN (2001), o, más recientemente WriGHT, SPARKS y O'HAIR (2008)-. El interés por el estudio de las enfermedades raras, desde una perspectiva comunicativa, es bastante más limitado. Así, podemos consultar el estudio de RIDAO (en prensa) sobre la representación discursiva de la locución "enfermedades raras" en el diario español El País. Según los datos que disponemos, son más frecuentes las investigaciones que abordan patologías concretas, como el síndrome de Williams (GARAYZÁBAL, 2004), el síndrome de Asperger (RodríGueZ MuÑoz 2007 y 
2010 o Rodríguez Muñoz, Ridao y ReQuena 2010) o el síndrome de Guilles de la Tourette (FÉRRIZ en prensa), por citar algunos ejemplos de los escasos estudios sobre enfermedades raras con un enfoque comunicativo.

\section{Marco teórico}

En la actualidad, las investigaciones del lenguaje asiduamente se acotan a la llamada lingüistica de la comunicación, pues se ha superado una primera fase de paradigma tradicional y una segunda etapa de paradigma estructural-generativista (GUTIÉRREZ ORDÓÑEZ, 2002). Será en la segunda mitad del siglo XX cuando nazcan distintas disciplinas en el ámbito de la lingüística, como es el caso de la pragmática, el análisis conversacional, la lingüística del texto, e incluso el análisis del discurso; en este último caso, destacamos la vertiente crítica. Estas relativamente nuevas disciplinas suelen presentar problemas tanto en la delimitación de sus ámbitos de estudio, así como en la existencia de una metodología de trabajo comúnmente aceptada por la mayoría de sus adeptos.

De otra parte, numerosos investigadores se han mostrado reacios a la hora de ejecutar investigaciones de índole multidisciplinar, si bien en las últimas décadas este panorama está cambiando. De esta manera, LAKOFF (2003) apoya explícitamente los estudios de naturaleza interdisciplinar, más en concreto hace alusión a los distintos enfoques con los que deben trabajar los expertos en análisis del discurso. En nuestra opinión, a estas alturas encontramos una postura casi unánime por parte de los investigadores para admitir la viabilidad y, sobre todo, la necesidad del estudio conjunto de disciplinas que tradicionalmente han estado inconexas. Sin ir más lejos, un ejemplo de ello es el modesto artículo que estamos presentando. Es evidente que nadie duda de la dificultad añadida que presentan los proyectos transdisciplinares.

En esta ocasión, nos decantamos por abordar el marco teórico desde un enfoque metodológico interdisciplinar, como es el análisis crítico del discurso. Para acotar sus competencias, ofrecemos unas conocidísimas palabras textuales de VAN DIJK que están recogidas en el artículo titulado "Principles of Critical Discouse Analysis", donde -a nuestro juicio- sienta las bases de esta corriente investigadora:

"Although there are many directions in the study and critique of social inequal-

ity, the way we approach these questions and dimensions is by focusing on the role of discourse in the (re)production and challenge of dominance. Dominance is defined here as the exercise of social power by elites, institutions or groups, that results in social inequality, including political, cultural, class, ethnic, racial and gender inequality. This reproduction process may involve such different 'modes' of discoursepower relations as the more or less direct or even support, enactment, representation, legitimation, denial, mitigation or concealment of dominance, among others. More specifically, critical discourse analysis want to know what structures, strategies or other properties of text, talk, verbal interaction or communicative events play a role in these modes of reproduction" (VAN DIJK, 1993: 249-250).

En consonancia, MARTín Rojo (2000: 115) nos recuerda que el análisis crítico del discurso interpreta que los discursos son prácticas sociales, en el sentido de que la producción y los efectos de la misma quedan regulados socialmente y que "el propio 
análisis tiene consecuencias e implicaciones tanto sobre la producción de los discursos como sobre su recepción, pero también sobre las condiciones sociales en las que estos discursos emergen".

Debemos asumir que las ideologías que transmitimos a través de la comunicación controlan el conocimiento, $y$, además, resultan determinantes para influir en las opiniones de los individuos sobre dicho acontecimiento (VAN DIJK, 1998). En este aspecto tienen especial incidencia los medios de comunicación, puesto que en la sociedad actual las personas somos asiduas consumidoras de las informaciones emitidas por tales medios. Si centramos nuestro campo de estudio en el análisis crítico del discurso aplicado a la prensa, van Dijk defiende la adaptación concreta a este medio de comunicación:

"Los informes periodísticos de la prensa pertenecen a una familia de tipos de textos informativos que necesitan su propio análisis estructural. Es decir, deben clarificarse las propiedades generales del discurso que desarrollan y las estructuras más específicas o características que los distinguen de otros textos de los medios de comunicación o textos similares diferentes a estos últimos, como los relatos" (VAN DiJK, 1990: 251).

En nuestra opinión, para trabajar la dimensión cualitativa de la representación discursiva, creemos que podría resultar interesante proponer una metodología de trabajo que fusione los principios que establece el análisis crítico del discurso con la semiótica. No olvidemos que esta disciplina dedicada al estudio de los signos en la vida social suele denominarse también semiología, y en ella destacan obras tan relevantes como Barthes (1967), Eco (1976), Hodge y Kress (1988), o ChANDler (2002). Si entendemos que los procesos de comunicación que se establecen en la sociedad -en este estudio aludimos a comunicación unidireccional, pues no existe retroalimentación directa en prensa- pueden respaldarse en acciones (tanto en su vertiente positiva como negativa), se podría establecer una clasificación de posibilidades.

Desde la perspectiva semiótica trabajaremos con las variables HACER y NO HACER combinadas con QUERER, NO QUERER, PODER, NO PODER, SABER Y NO SABER. Esta armonía de categorías semióticas da lugar a una serie de tipologías discursivas, las cuales presentamos en la siguiente tabla. En cuanto a DECIR y NO DECIR -variables muy utilizadas en semiótica- hemos preferido obviarlas, como consecuencia de que hablar de un proyecto no siempre implica que culmine haciéndose; por ende, le otorgamos más relevancia al HACER que al DECIR, siendo conscientes de que la relación existente entre estas dos últimas variables citadas es muy estrecha. He aquí el esquema anunciado:

\begin{tabular}{|l|l|}
\hline HACER/QUERER ------------- Compromiso & NO HACER/QUERER ---------- Preocupación \\
\hline HACER/NO QUERER ---------- Continencia & NO HACER/NO QUERER ------ Indiferencia \\
\hline HACER/PODER --------------- Consolidación & NO HACER/PODER ----------- Abstinencia \\
\hline HACER/NO PODER ---------- Reivindicación & NO HACER/NO PODER ------- Impotencia \\
\hline HACER/SABER --------------- Aseveración & NO HACER/SABER ---------- Inhibición \\
\hline HACER/NO SABER ----------- Obcecación & NO HACER/NO SABER ------- Prevención \\
\hline
\end{tabular}


Este marco teórico puede ser utilizado para analizar corpus de muy distinta naturaleza. Así pues, en nuestro caso, al tener una línea de investigación dedicada al discurso periodístico, en varias ocasiones -FÉRRIZ y RIDAO (2008) o RIDAO (2010)- hemos realizado publicaciones sobre la imagen de la inmigración en prensa, utilizando como método de investigación las propuestas del análisis crítico del discurso y las categorías que ofrece la semiótica.

\section{Corpus y metodología}

El corpus con el que trabajamos ha sido extraído de las bases de datos digitales que ofrecen distintos periódicos españoles. En particular, nos hemos centrado en los tres diarios que presentan mayor tirada nacional; esto es, El País, El Mundo y ABC. La etapa cronológica seleccionada comprende desde el inicio de tales periódicos hasta el 31 de diciembre de 2009. El concepto de búsqueda, con criterio de aparición en todos los campos, ha sido, lógicamente, ataxia. En total hemos encontrado noventa y siete noticias que recogen la palabra ataxia. Concretamente, de El País hemos recopilado cuarenta, de $E l$ Mundo treinta y nueve y de $A B C$ dieciocho.

En cuanto a la metodología, por un lado, nos centraremos en observar las dimensiones cuantitativas; o sea, datos relacionados con el número de noticias encontradas en que aparece el término ataxia en función del diario y del año en que es publicado. Partiremos de un gráfico donde observamos la distribución del corpus según los diarios donde han sido publicadas las noticias, para analizar, en el siguiente diagrama, datos más concretos sobre la edición. En cuanto al perfil cualitativo del presente estudio, partiendo de una selección de fragmentos extraídos del corpus, nos basaremos en un análisis discursivo en el que combinamos las propuestas teóricas del análisis crítico del discurso con los principios sobre los que se asienta la semiótica, a la vez que aportamos información relacionada con la temática del corpus y haremos especial hincapié en los discursos emitidos por los distintos grupos de actores.

\section{Resultados}

\section{1. Datos cuantitativos}

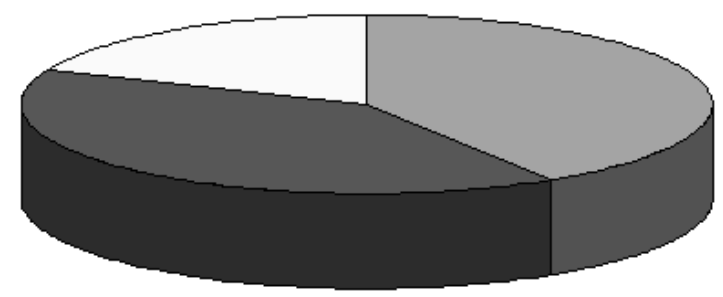

EI Pais $\square$ El Mundo $\square A B C$

En total hemos encontrado noventa y siete noticias en los tres diarios de mayor tirada en España. En este gráfico ofrecemos la distribución de tales publicaciones, cuyos porcentajes son el $41.2 \%$ de El País (cuarenta en números reales), el $40.2 \%$ de $E l$ Mundo (treinta y nueve en números reales) y el $18.5 \%$ de $A B C$ (dieciocho en números reales):

En este otro diagrama observamos las noticias aparecidas en prensa en función del diario, las variables cronológicas del año de edición y el número: 


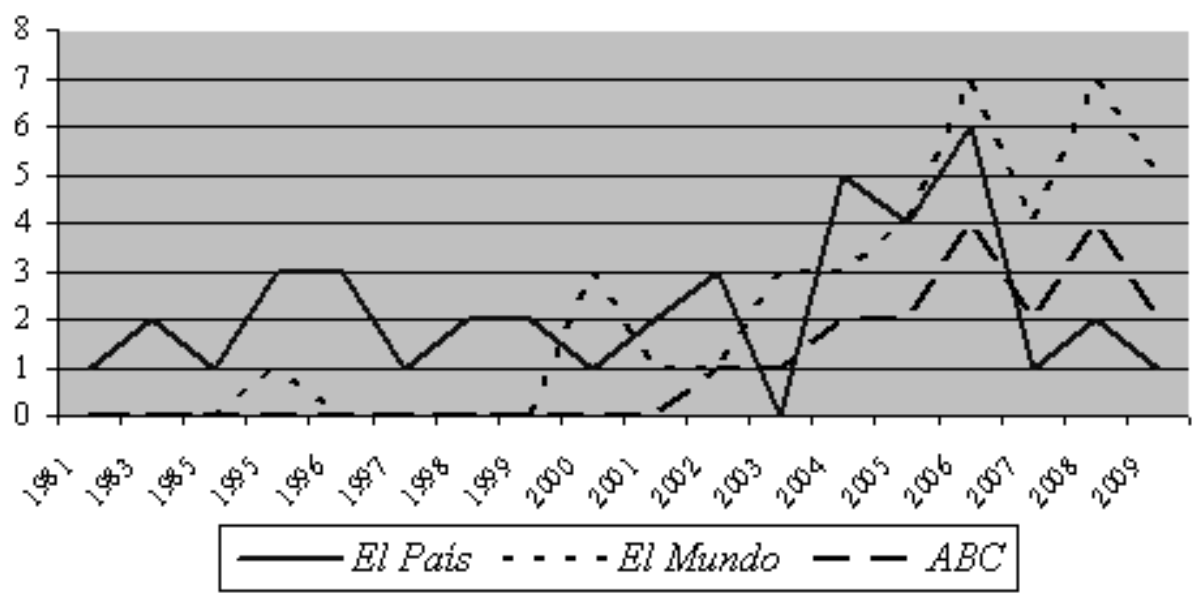

Como podemos comprobar, el diario El País es el primero que incluye el término ataxia, concretamente en 1981. Sin embargo, hay que esperar hasta 1995 para poder leer este vocablo en El Mundo y hasta 2002 para encontrarlo en $A B C$. No obstante, si comparamos el número de noticias que hallamos en El País y El Mundo, nos daremos cuenta de que las cifras con muy parecidas (recordemos, cuarenta y treinta y nueve en números reales respectivamente). Esto indica que a pesar de que El Mundo haya agregado entre sus páginas noticias relacionadas con ataxia mucho más tarde que El País, concretamente catorce años después, en la primera década del siglo XXI corroboramos que el número de publicaciones aparecidas en El Mundo que aborda esta enfermedad rara es bastante más elevado que el de El País.

En la siguiente tabla mostramos las cifras reales y su representación estadística en función de si las noticias donde leemos la voz ataxia tratan temas relacionados con el ámbito médico o no. Además, analizamos si abordan de manera prioritaria el tema de la patología de la ataxia, o bien esta enfermedad tiene una relevancia de segundo orden. Por supuesto, detallamos los datos según diarios, a la vez que facilitamos estadísticas globales.

\begin{tabular}{|c|c|c|c|c|c|c|c|c|c|}
\hline \multicolumn{2}{|c|}{} & \multicolumn{2}{|c|}{ EI País } & \multicolumn{2}{c|}{ El Mundo } & \multicolumn{2}{c|}{ ABC } & \multicolumn{2}{c|}{ Datos globales } \\
\cline { 2 - 10 } & \multicolumn{2}{|c|}{$\begin{array}{c}\text { Cifras } \\
\text { reales }\end{array}$} & $\%$ & $\begin{array}{c}\text { Cifras } \\
\text { reales }\end{array}$ & $\%$ & $\begin{array}{c}\text { Cifras } \\
\text { reales }\end{array}$ & $\%$ & $\begin{array}{c}\text { Cifras } \\
\text { reales }\end{array}$ & $\%$ \\
\hline $\begin{array}{c}\text { Relación te- } \\
\text { mática con } \\
\text { salud }\end{array}$ & Sí & 38 & $95 \%$ & 36 & $92.3 \%$ & 16 & $88.8 \%$ & 90 & $92.7 \%$ \\
\cline { 2 - 10 } & No & 2 & $5 \%$ & 3 & $7.6 \%$ & 2 & $11.1 \%$ & 7 & $7.2 \%$ \\
\hline $\begin{array}{c}\text { Ataxia } \\
\text { como tema } \\
\text { principal }\end{array}$ & Sí & 17 & $42.5 \%$ & 13 & $33.3 \%$ & 5 & $27.7 \%$ & 35 & $36.08 \%$ \\
\cline { 2 - 10 } & No & 23 & $57.5 \%$ & 26 & $66.6 \%$ & 13 & $72.2 \%$ & 62 & $63.9 \%$ \\
\hline
\end{tabular}




\section{2. Datos cualitativos}

A continuación, recogemos una muestra significativa (entendiendo por tal también la proporcionalidad) del contenido que podemos leer en nuestro corpus sobre el discurso emitido en torno a la patología de la ataxia. Como es lógico, en este apartado nos hemos centrado en aquellas noticias cuyo contenido se centra principalmente esta en enfermedad.

\section{El País}

-«Soy una madre residente en Prado del Rey (Cádiz) y quisiera hacer una llamada a todos los que como yo tienen hijos afectados de ataxia, a fin de que pongamos nuestros esfuerzos en común y conseguir siquiera sea un algo de esperanza para todos. Resulta desalentador que no exista en España ninguna asociación que represente y proteja a esas criaturas, como ocurre en muchos casos, sí las haya en otros países [...]» $(23 / 08 / 1983)$.

- «Soy un afectado de ataxia de Friedreich que quisiera aprovechar la tribuna de su periódico para hacer una llamada de atención sobre este tipo de enfermedades tan terribles como desconocidas, primero a los propios afectados y luego a la sociedad en general. [...] Como he dicho antes, la medicina no ofrece ningún tratamiento. ¿Y las instituciones? ¿Y la sociedad?... Ignorancia. Fuera, por lo menos, se está investigando. Aquí, nada. Lo dicho para la ataxia de Friedreich es sustancialmente válido para todas las ataxias hereditarias. Pues bien, unos pocos enfermos nos hemos encontrado ipor fin! Y pretendemos constituir una asociación que nos ayude a romper este aislamiento. Ésta es una llamada sobre todo para los afectados de ataxia. Tenemos que unirnos y ayudarnos a nosotros mismos; pero también a todas las personas e instituciones que puedan y quieran prestarnos su ayuda» (08/01/1985).

-«'En el Instituto Cajal', dice, 'donde llevamos cinco años estudiando las bases moleculares de este grupo de patologías neurodegenerativas, hemos obtenido resultados muy alentadores en modelos animales. El objetivo es ir hacia terapias combinatorias por tres vertientes: la farmacológica, la terapia celular y la terapia génica’» $(25 / 06 / 2002)$.

-«"Hace cuatro años reformaron el pasadizo, levantaron el empedrado. Pedí por escrito a la junta municipal una rampa. Me contestaron que no había presupuesto. Al mes volvieron, se habían olvidado de poner unos cables para las farolas. Abrieron todo otra vez. Volví a pedir la rampa. Ni contestaron. Para las farolas hubo presupuesto, para nosotros no", relata Miguel Ángel con una amargura difícil de disimular. [...] "Bastaría un director general, o un concejal. Sólo el que está como nosotros es capaz de comprenderlo", explica Díaz, miembro de la Asociación Española de Enfermedades Musculares [...] En países como Dinamarca, Miguel Ángel y Javier podrían vivir por sí mismos gracias a las ayudas estatales. En España no. "Con una pensión de 390 euros cada uno al mes. ¿Dónde vamos?”, se pregunta Miguel Ángel. Su madre asiente. Les mira como se mira a dos niños de 11 años. Es la única que se atreve a hacer la última pregunta: “¿Qué va a pasarles cuando yo no esté?”》(01/03/2004). 
-«"Yo sólo tuve 20 sesiones de fisioterapia financiados por la sanidad pública en mi vida", lamentó. También solicitó a la Xunta que cree una unidad de referencia de ataxia en Galicia. Concretamente reivindicó que esté en el CHUS, ya que los doctores de este centro son los que han desarrollado esta investigación y "visto a más pacientes". "Al ser una enfermedad tan extraña muchos neurólogos no están acostumbrados a tratarnos", añadió. Manuel Arias respondió que sería "perfectamente asumible", y señaló que tendría que ser la Consellería de Sanidade la que debe evaluar la situación y decidir su ubicación en el caso de que se crease» $(17 / 12 / 2009)$.

\section{El Mundo}

-«El que aparezca un gen cada semana, y sus descubridores quieran rentabilizar con publicidad haber estado años de trabajo tras una determinada secuencia de ADN, es comprensible. Sin embargo, muchos de los hallazgos que se «venden» a bombo y a platillo tienen, de momento, pocos efectos prácticos» (29/06/1995).

-«Quisiera saber, si es posible, si existe algún tratamiento, aunque según lo que me han explicado no hay $(05 / 12 / 2005) »$.

-«No quiere vivir con la idea del «futuro decidido por la enfermedad» que le transmitieron en su diagnóstico. [...] Soy realista, esta enfermedad es muy dura, no lo puede negar nadie, y tienes que esforzarte mucho física y emocionalmente, también tus amigos y tu familia. Tampoco quiero dar la imagen de que no pasa nada, de chico 'superguay' con ataxia. Quiero dar a conocer la realidad, mi realidad, la de un joven que intenta llevar una vida normal» (20/05/2006).

-«Tengo 65 años y desde los 18 padezco ataxia espinocerebelosa. Desde hace dos años estoy en silla de ruedas. El médico de la seguridad social me ve cada seis meses, un año o año y medio. El único tratamiento que tengo desde hace años es $2.700 \mathrm{mg}$, en tres tomas, de Neurontin. Creo que este medicanto (sic) no me sirve ya para nada y me está perjudicando. ¿Cómo puedo dejar el medicamento? ¿Y si hay algún otro tratamiento?» $(21 / 08 / 2006)$.

-«En mi familia se padece un tipo de ataxia SCA7. Me gustaría saber los posibles tratamientos. ¿Podría orientarme de algún centro donde puedan recibir rehabilitación neurológica?» (27/12/2007).

\section{$A B C$}

-«A ello se refirió la directora general de los discapacitados para la igualdad y el bienestar social de la Junta de Andalucía, que especificó que «como representante de la Consejería no me puedo quedar sólo con la reivindicación, sino que tenemos que trasladar las propuestas en medidas concretas y avances que tienen que venir a sumarse a ese camino importante que la asociación lleva realizando»"» (23/09/2004). 
$-\ll$ Sin embargo, estas personas, tan necesitadas de apoyo, no tienen derecho a un espacio que se les promete lleno de ayudas. ¿Dónde está la labor del tan cacareado Ministerio de Igualdad, que apoya a los colectivos más desprotegidos? ¿Por qué las enfermedades «raras» no tienen ni el apoyo ni el soporte que este Ministerio destina a actividades como el Día de la Bicicleta o el Orgullo Gay? ¿Dónde la famosa discriminación positiva de los más necesitados? ¿Dónde las prometidas ayudas de accesibilidad en edificios y espacios públicos? Es cierto que algunas instituciones, cuando conocen esta cruda realidad, apoyan con sus escasos medios, pero la falta de información y el exceso de demagogia impiden ampliar este soporte a organismos estatales más poderosos. Somos una sociedad hipócrita, y la grandeza es que la respuesta que nos dan es una profunda mirada cargada de tristeza y cariño» $(01 / 10 / 2009)$.

\section{Discusión}

\section{1. Datos cuantitativos}

Los periódicos El País y El Mundo son los que publican las cifras más elevadas de noticias donde aparece el término ataxia. En concreto, cada uno de ellos suma aproximadamente dos quintas partes del corpus con el que estamos trabajando. Por ende, las publicaciones aparecidas en $A B C$ tan solo representan una quinta parte del total. En cuanto a los años de edición, El País fue el pionero en abordar esta cuestión en un artículo fechado en 1981. Durante dicha década este diario da a conocer tres noticias más relacionadas con ataxia. En los años noventa El País sigue su tendencia de tratar este tema, más exactamente edita once noticias. Por estas fechas, El Mundo debuta en la incorporación de información sobre ataxia, si bien la inmensa mayoría de estas aportaciones las leemos en lo que llevamos de siglo XXI. $A B C$ es el último diario en incluir entre sus páginas aspectos relacionados con esta patología poco prevalente, iniciando esta tendencia en 2002. El año en que El País recoge mayor número de noticias sobre ataxia es 2006, con la aparición de seis artículos. Por su parte, en El Mundo observamos que en 2006 y 2008 ofrece siete noticias sobre el tema en cuestión. $\mathrm{Cu}$ riosamente, estas fechas coinciden con los datos que tenemos de $A B C$, aunque este último diario presenta cuatro publicaciones por año. De todo ello deducimos que 2006 es la etapa en que podemos leer más información relacionada con ataxia, pues suma entre los tres diarios un total de diecisiete noticias. El segundo lugar de esta lista lo ocupa el año 2008, contando con trece referencias.

En El País constatamos que todas las noticias donde vemos el vocablo ataxia tratan temas relacionados con el ámbito propio de la medicina, a excepción de dos: la primera de ellas versa sobre producción cinematográfica («La Gran Vía es más cinematográfica que las calles de Nueva York») y la segunda cuenta la historia de un enfermo de ataxia que relata la ayuda que ha obtenido en Internet para recabar información sobre su dolencia («¿Cómo ha cambiado tu vida Internet?»). De otro lado, las referencias publicadas en El País donde la ataxia aparece como tema principal ascienden, en números reales, a diecisiete (un $42.5 \%$ ), frente a veintitrés noticias (un 
57.5\%) donde encontramos el término ataxia aunque esta enfermedad no sea la cuestión más relevante, sino que constituye un tema secundario.

En El Mundo también hallamos un alto predominio de escritos donde leemos la voz ataxia relacionada con informaciones médicas. La cifra real de excepciones suma tres publicaciones, comunicando todas ellas el suceso del hallazgo de un cuerpo muerto en Valladolid en 2008. En cuanto a los porcentajes relativos al número de noticias que enfoca la ataxia como tema principal, observamos que es inferior con respecto a las cifras que manejamos de El País, representando en el caso de El Mundo el 33.3\% (trece en números reales), lo que implica que el $66.6 \%$ (veintiséis en números reales) responde a noticias donde ataxia no supone el grueso de la información.

Con respecto a $A B C$, señalamos que -en sintonía con los otros dos diarios analizados- apuesta por un claro predominio de incorporar el término ataxia en noticias vinculadas con el campo de la sanidad. Las dos excepciones halladas responden a los siguientes titulares: «La vivienda y el bolero de Ravel» y «La Guardia Civil confirma que los restos hallados en 2006 son de la desaparecida de Laguna»; en el primer artículo citado se reflexiona sobre la subida del precio de la vivienda, mientras que el segundo habla del suceso de Valladolid del que también se ha hecho eco el diario El Mundo (como comentábamos en el párrafo anterior). En cambio, al analizar las estadísticas macroestructurales relacionadas con el tema principal de las informaciones, constatamos que tan solo cinco noticias (lo que representa un 27.7\%) versan sobre ataxia; por tanto, hay trece (el $72.2 \%$ ) que incluyen el vocablo sometido a estudio, si bien no exponen esta patología de manera prioritaria.

\section{2. Datos cualitativos}

Haciendo un análisis del contenido de las noticias que abordan la enfermedad de la ataxia, nos daremos cuenta de que El País es el diario que recoge con mayor asiduidad las palabras de actores implicados en esta patología poco prevalente, entendiendo por tales a afectados, familiares e incluso personal investigador y autoridades políticas. Estos cuatro grupos apuestan por el compromiso, en el sentido de que muestran una actitud firme de QUERER HACER. Como era de esperar, tanto los propios enfermos como sus familiares suelen hacer uso de un discurso reivindicativo, puesto que la imagen que transmiten es claramente una combinación de HACER y NO PODER. En el siguiente ejemplo que aportamos observamos que la posición del afectado por ataxia es muy reivindicativa, al tiempo que justifica estos argumentos con el hecho de que la sanidad de otras naciones financia investigaciones de dicha índole: « ¿Y las instituciones? ¿Y la sociedad?... Ignorancia. Fuera, por lo menos, se está investigando. Aquí, nada» (08/01/1985). En este otro caso, el paciente, al hablar de las dificultades de acceso por la vía y los espacios públicos para las personas que se encuentran en sillas de ruedas, pone en evidencia la nula empatía de las elites políticas hacia este colectivo desfavorecido: «Bastaría un director general, o un concejal. Sólo el que está como nosotros es capaz de comprenderlo» (01/03/2004).

Junto a ello, debemos señalar que el grupo de afectados y familiares de los mismos también hace gala de un discurso de consolidación, teniendo en cuenta que la reivindicación está presente en mayor grado. Retomando el tema del discurso consolida- 
dor, hallamos un rotundo HACER y PODER al tratar aspectos de la creación de asociaciones; instituciones que no solo facilitan información a nuevos pacientes, sino que en la actualidad estos organismos actúan como plataformas para pedir los derechos que estos enfermos merecen. Desde otro enfoque, las palabras emitidas por los investigadores se centran en comentar los avances alcanzados en los estudios científicos. Por tanto, podríamos decir que este colectivo presenta un HACER y un SABER; esto es, un discurso de aseveración. Sobre ello, conviene recordar que las investigaciones requieren mucho tiempo, y que los descubrimientos de estos profesionales a menudo implican mejoras en la calidad de vida de los pacientes de manera muy limitada.

En cuanto a El Mundo, destacamos que en una noticia publicada en 1995 el autor invita a los lectores a reflexionar sobre la cautela interpretativa que debemos seguir cuando nos informan del descubrimiento de determinados avances científicos, advirtiendo que con frecuencia tales innovaciones carecen de un enfoque práctico generalizado. Las voces emitidas por los actores implicados abogan por un discurso de compromiso, debido a que optan por un QUERER HACER. Subrayamos que buena parte de los enunciados emitidos por afectados de ataxia publicados por este diario se centre en la petición de información sobre esta patología poco prevalente. Del mismo modo, podemos encontrar relatos sobre el día a día de estos pacientes y la postura emocional adoptada: «Tampoco quiero dar la imagen de que no pasa nada, de chico 'superguay' con ataxia. Quiero dar a conocer la realidad, mi realidad, la de un joven que intenta llevar una vida normal» (20/05/2006).

En $A B C$ leemos unas aportaciones de personal de administración y representación política. En consonancia con este aspecto, en RIDAO (en prensa) comprobábamos que este grupo de actores refleja en menor medida su discurso sobre las enfermedades raras en los medios de comunicación. Volviendo con el corpus sobre la ataxia, observamos que este personaje político aboga no solo por un QUERER HACER (recordemos, discurso comprometido), sino que de sus palabras interpretamos un HACER y un PODER, es decir, un discurso de consolidación: «como representante de la Consejería no me puedo quedar sólo con la reivindicación, sino que tenemos que trasladar las propuestas en medidas concretas y avances que tienen que venir a sumarse a ese camino importante que la asociación lleva realizando» (23/09/2004). En otro fragmento extraído de $A B C$ hallamos una postura muy crítica hacia la indiferencia mostrada -según el autor de la carta- por parte de las autoridades políticas a los enfermos con ataxia. En dicho discurso interpretamos reivindicación (HACER y NO PODER) e impotencia (NO HACER y NO PODER). Cerramos este epígrafe recogiendo unas palabras que entendemos que muestran un discurso desesperado y, por ende, descortés, al romper con la actitud políticamente correcta desde la que solemos cifrar nuestros mensajes: «Somos una sociedad hipócrita, y la grandeza es que la respuesta que nos dan es una profunda mirada cargada de tristeza y cariño» $(01 / 10 / 2009)$.

\section{Conclusiones}

1. La invisibilidad ha caracterizado a las patologías poco prevalentes hasta finales del siglo XX en España. Con la creación de FEDER (Federación Española de Enfermedades Raras) esta situación está cambiando. No obstante, a pesar de que con mediana 
frecuencia vemos en los medios información sobre estas patologías, la población, en general, desconoce su existencia.

2. La poca repercusión que han presentado las patologías poco prevalentes guarda relación directa con la escasez de publicaciones que tienen como foco de estudio estas enfermedades. La mayoría de tales trabajos parte de una perspectiva principalmente médica, si bien en el campo de la comunicación podemos encontrar estudios que toman como base tanto el discurso patológico como el discurso sobre estas patologías poco frecuentes.

3. En la actualidad, son muchos los investigadores que se decantan por abordar trabajos de naturaleza interdisciplinar. Sobre esta base se asienta el análisis crítico del discurso, el cual es aplicado para analizar textos de muy diversa índole. En este caso estamos indagando en un corpus periodístico relacionado con la enfermedad de la ataxia, apoyándonos también en bases teóricas ofrecidas por la semiótica.

4. De los tres periódicos sometidos a análisis, El País presenta la cifra más elevada de noticias que recogen la voz ataxia (cuarenta en números reales), seguido de $E l$ Mundo (treinta y nueve) y $A B C$ (dieciocho). Es en 2006 cuando vemos publicadas más noticias con el vocablo ataxia. Tan solo en el $36.08 \%$ del corpus obtenido la ataxia es el centro temático, frente al $63.9 \%$ cuyo contenido suele informar de asuntos sanitarios en general.

5. El discurso de los actores implicados en enfermedades raras que encontramos en este corpus periodístico se caracteriza por un marcado predominio del compromiso; esto es, emplea las variables HACER y QUERER. El grupo que aparece con mayor asiduidad es el de enfermos, con un discurso reivindicativo al presentar un panorama de HACER y NO PODER. Con las mismas tipologías semióticas encontramos testimonios de familiares afectados por ataxia. Por su parte, los investigadores apuestan por un HACER y un SABER, lo que implica aseveración. Por último, la representación política sostiene un discurso de consolidación (HACER y PODER).

\section{Referencias bibliográficas}

AUTORES VARIOS (2009): Enfermedades raras. Manual de humanidad para encontrar las claves de la felicidad. Madrid, Loquenoexiste.

BARTHES, Roland (1967): Elements of Semiology. London, Jonathan Cape.

CHANDLER, Daniel (2002): Semiotics: The Basics. London, Routledge.

ECO, Umberto (1976): A Theory of Semiotics. London, Macmillan.

ENSERio (2009): Estudio sobre Situación de Necesidades Sociosanitarias de las Personas con Enfermedades Raras en España. Madrid, FEDER/Caja Madrid.

FÉRRIZ, María (en prensa): "Desarrollo de destrezas comunicativas no verbales en clases de L1 para alumnos con síndrome de Guilles de la Tourette", en Actas del XXVII AESLA Conference.

FÉRRIZ, María y RIDAO, Susana (2008): "Estudio discursivo de la inmigración en los debates españoles sobre el Estado de la Nación de 2003 y 205", en Discurso \& Sociedad, no 2 (4), pp. 716-745. 
GARAYZÁBAL, Elena (2004): “Cantar 'a capela' es cantar en español: el manejo del léxico en el síndrome de Williams", en Interlingüistica, no 15, pp. 573-582.

GONZÁLEZ-MENESES LÓPEZ, Antonio et al. (2008): Plan de atención a personas afectadas por enfermedades raras. Sevilla, Consejería de Salud de la Junta de Andalucía.

GUTIÉRREZ ORDÓÑEZ, Salvador (2002): De pragmática y semántica. Madrid, Arco/Libros.

GWYN, Richard (2001): Communicating Health and Illness. London, Sage.

HODGE, Robert y KRESS, Gunther (1988). Social Semiotics. Ithaca, Cornell UP.

LAKOFF, Robin (2003): "Nine Ways of Looking at Apologies: The Necessity for Interdisciplinary Theory and Method in Discourse Analysis", en SCHIFFRIN, Deborah, TANNEN, Deborah y HAMILTON, Heidi: The Handbook of Discourse Analysis. Oxford, Blackwell Publishing, pp. 199-214.

LUCY, Yardley (1997): Material Discourses of Health and Illness. London, Routledge.

MARTÍN ROJO, Luisa (2000): "Enfrentamiento y consenso en los debates parlamentarios sobre la política de inmigración en España", en Oralia, n 3, pp. 113-148.

MATEO, Virginia (2001): Enfermedades Raras: Situación y demandas sociosanitarias. Madrid, Instituto de Migraciones.

OBSERVATORIO DE LA DISCAPACIDAD (2002): Ataxias hereditarias. Guía de familia. Madrid, Observatorio de la Discapacidad.

RIDAO, Susana (2010): "La regularización de inmigrantes de 2004: Análisis discursivo de los grupos parlamentarios en el Debate sobre el Estado de la Nación de 2005”, en Sociedad y Discurso, n ${ }^{\circ}$ 17, pp. 21-43.

RIDAO, Susana (en prensa): "Enfermedades raras y discurso. Análisis de las noticias publicadas en el diario El País". En FORNIELES, Javier et alii: Comunicación y salud. San Sebastián, Gakoa.

RODRÍGUEZ MUÑOZ, Francisco José (2007): "Comunicación, autismo y síndrome de Asperger. Hacia un estado de la cuestión desde el punto de vista bibliográfico", en Tonos Digital, $\mathrm{n}^{\circ}$ 13, pp. 1-37.

RODRÍGUEZ MUÑOZ, Francisco José (2010): Síndrome de Asperger. Aproximación pragmalingüística y materiales. Valencia, Servicio de Publicaciones de la Universidad de Valencia.

RODRÍGUEZ MUÑOZ, Francisco José; RIDAO, Susana y REQUENA, Samantha (2010): "Diseño de un curso sobre habilidades comunicativas en plataformas de enseñanza virtual: El síndrome de Asperger", en CASTRO, Ángel y GUILLÉN-RIQUELME, Alejandro: Evaluación de la calidad de la educación superior y de la investigación. Granada, AEPC, pp. 206-210.

SONTAG, Susan (1978): Illness as Metaphor. New York, Straus \& Giroux. 
VAN DIJK, Teun A. (1990): La noticia como discurso. Comprensión, estructura y producción de la información. Barcelona, Paidós.

VAN DIJK, Teun A. (1998): Ideology. A Multidisciplinary Approach. London, Sage.

VAN DIJK, Teun A. (1993): "Principles of Critical Discourse Análisis", en Discourse \& Society, $\mathrm{n}^{\circ}$ 4(2), pp. 249-283.

WRIGHT, Kevin, SPARKS, Lisa y O'HAIR, Dan (2008): Health Communication in the $21^{\text {st }}$ Century. Oxford, Blackwell. 